\title{
ANÁLISE DO GRAU DE ORIENTAÇÃO PARA OS STAKEHOLDERS DAS EMPRESAS DE CAPITAL ABERTO DA REGIÃO SUL DO BRASIL
}

\author{
Renato Fabiano Cintra ${ }^{1}$ \\ Wagner Seiki Oguido ${ }^{2}$ \\ Antonio Oliveira de Carvalho ${ }^{3}$ \\ Saulo Fabiano Amâncio-Vieira ${ }^{4}$
}

\begin{abstract}
RESUMO: Trata-se de uma replicação adaptada do artigo Developing a Scale for Stakeholder Orientation, pois objetivou analisar o grau de orientação para stakeholders das empresas de capital aberto da região sul do Brasil. Logo quatro categorias de stakeholders primários foram selecionadas: clientes, concorrentes, acionistas e colaboradores. Aplicou questionário com questões fechadas (escala de Likert). Realizou análise de correlação entre os itens dos questionários com seus stakeholders. Os resultados apresentam semelhança com o estudo original, que pode ser considerada uma tendência das empresas de capital aberto, porém não pode ser confirmada de que há correlação entre o grau de orientação para stakeholders e desempenho da empresa. Mesmo assim, pode evidenciar que existem correlação e alinhamento dos itens, embora tenham abordagem diferentes.
\end{abstract}

Palavras-chave: Estratégia. Stakeholder. Orientação para Stakeholders. Desempenho da Empresa. Empresa de Capital Aberto.

\footnotetext{
${ }^{1}$ Mestre em Administração pelo PPGA-UEL/PR (2013), Doutorando em Administração pelo PPGAUNINOVE/SP (2014) e Funcionário Público Federal - Administrador (2012). Universidade Federal da Grande Dourados (UFGD/MS) e Universidade Estadual de Londrina (UEL/PR). Brasil. E-mail: renatocintra@hotmail.com

${ }^{2}$ Graduado em administração pela Universidade Estadual de Londrina (UEL/PR). Universidade Estadual de Londrina (UEL/PR). Paraná. Brasil. E-mail: wagner oguido@yahoo.com.br

${ }^{3}$ Doutorando em administração pela Universidade Nove de Julho (UNINOVE). Mestre em administração pela Universidade Salvador (UNIFACS). Centro Universitário Jorge Amado UNIJORGE. Bahia. Brasil. E-mail: professorcarvalho@rocketmail.com

${ }^{4}$ Doutor em Administração pelo PPGA/UNINOVE/SP e professor do Programa de Pós-Graduação em Administração da Universidade Estadual de Londrina (PPGA/UEL). Coordenador do curso de Administração. Universidade Estadual de Londrina (UEL/PR). Paraná. Brasil. E-mail: saulo@uel.br
}

Ágora: R. Divulg. Cient., v. 21, n. 2, p. 55-75, jul./dez. 2016 (ISSNe 2237-9010) 


\section{ANALYSIS OF THE DEGREE OF GUIDANCE FOR THE STAKEHOLDERSOF PUBLICLY TRADED COMPANIES FROM SOUTHERN REGION OF BRAZIL}

ABSTRACT: This is an adapted replication paper Developing a Scale for Stakeholder Orientation as it aimed to analyze the degree of guidance for stakeholders o public companies in southern Brazil. Soon four categories o primary stakeholders were selected: customer, competitors, shareholders and employees. Applied questionnaire with closed questions (Likert scale). Conducted correlation analysis between the items of the questionnaire with tis stakeholders. The results are similar to the original study, which can be considered a trend of public companies, but can't be comfirmed that there is a correlation between the degree of guidance for stakeholders and business performance. Nevertheless, evidence that there may correlation and alignment of the items, but have different approach.

Keywords: Strategy. Stakeholder. Guidance for Stakeholders. Company performance. Publicly Traded Company.

\section{INTRODUÇÃO}

Vários autores, ao longo da história, buscaram apresentar uma definição do termo stakeholder (MILES, 2015). As diversas definições de stakeholders apresentadas normalmente permitem esclarecer a conceituação em função dos seguintes aspectos:apontam o elo entre a organização e o stakeholders - por meio de um verbo de ligação comoafetar, impactar, influenciar ou exercer; e mostram a relação entre os participantes - pela utilização de verbos como responsabilizar, apoiar, depender, dar significado, arriscar algo de valor, investir, prejudicar, beneficiar ou expressar.

"A forma mais comum de classificação dos stakeholders é considerar grupos de pessoas com um relacionamento distindo com a empresa" (FRIEDMAN; MILES, 2006, p.13), os quais podem ser distribuídos em: acionistas, clientes, fornecedores, empregados e comunidades locais - visão restrita do termo stakeholder (CINTRA et al., 2015). O conceito de stakeholders, de forma mais ampla, representa uma redefinição de todas as organizações, na medida em que determinam como estas devem ser conceituadas ou como deveriam ser (FRIEDMAN; MILES, 2006). Para 
Freeman (1984) a visão restrita do termo trata-se dos stakeholders primários e a visão ampla dos stakeholders secundários.

A definição recorrente foi descrita por Freeman (1984, p. 46), que stakeholder é "qualquer grupo ou indivíduo que pode afetar ou é afetado pela realização dos objetivos da empresa". Como teoria, os estudos de stakeholders têm se solidificado no campo da gestão organizacional e estratégia (CINTRA et al., 2014). De acordo com Laplume, Sonpar e Litz (2008) três períodos são evidentes no cenário internacional: incubação (1984-1991), crescimetno incremental (1991-1998) e maturidade da teoria (1999-presente).

Outra evolução foi o trabalho de Donaldson e Preston (1995), os quais mostram em seu artigo as diferenças entre o modelo corporativo convencional baseado na entrada e na saída de recursos e o modelo corporativo com base nos stakeholders, além de apresentar e explicar os três aspectos da teoria dos stakeholders: descritivo, instrumental e normativo. Destacam que no aspecto descritivo a empresa é vista como uma constelação de interesses corporativos e competitivos contendo valores intrínsecos, no instrumental examina as conexões ceteris paribus entre a prática de administração de stakeholder e a realização de vários objetivos do desempenho empresarial e no normativo tem-se a identificação dos guias morais e/ou filosóficos à administração das organizações (DONALDSON; PRESTON, 1995).

O trabalho foi delimitado como foco de estudo das seguintes categorias de stakeholders: clientes, concorrentes, colaboradores e acionistas, os quais são considerados por Donaldson e Preston (1995), Freeman (1984), Freeman et al. (2010), Friedman e Miles (2006) e Yau et al. (2007) como os grupos de stakeholders mais importantes para as empresas. Portanto, objetiva analisar o grau de orientação para os stakeholders das empresas de capital aberto da região sul do Brasil. Deste modo, a pesquisa visou, por meio da pesquisa quantitativa, verificar o grau de orientação para os stakeholders das empresas de capital aberto, e se a adoção dessa orientação para os stakeholders influencia no desempenho dessas empresas. O artigo está estruturado em: além da introdução, tem-se o suporte teórico voltado para a identificação dos stakeholders e orientação para os stakeholders. Na sequência são apresentados os procedimentos metodológicos. Na seção quatro 
tem-se a apresentação e análise dos resultados. E por fim, as considerações, limitações e orientações futuras ao campo científico.

\section{SUPORTE TEÓRICO}

Com intuito de apresentar o suporte teórico da presente pesquisa, optou por segmentar a seção em dois subitens: identificação dos stakeholders; e orientação para os stakeholders, como serão explanados na sequência.

\subsection{IDENTIFICAÇÃO DO STAKEHOLDER}

Existem diversas formas de identificar os stakeholders (ALMEIDA; FONTES FILHO; MARTINS, 2000; CINTRA; AMÂNCIO-VIEIRA; COSTA, 2014; FREEMAN, 1984; FREEMAN et al., 2010; FRIEDMAN; MILES, 2002; MITCHELL; AGLE; WOOD, 1997; REED et al., 2009). Partindo da definição de Freeman (1984) para stakeholders Mitchell, Agle e Wood (1997) apontam três atributos à identificação dos stakeholders, sendo: poder, legitimidade e urgência.

Em relação ao poder utilizam como definição as idéias de Webber(1947apud MITCHELL; AGLE; WOOD, 1997, p. 865) que afirma que poder é "a probabilidade de um agente em uma relação social estar em uma posição de realizar suas vontades apesar da resistência" e de Salancik e Pfeffer (1974, p. 3 apud MITCHELL; AGLE; WOOD, 1997, p. 865), para reconhecer o poder, em que é "a habilidade daqueles que possuem poder realizar aquilo que eles desejam". Os stakeholders em questão tem poder na relação quando podem obter ou têm meios coercivos, utilitários ou normativos para impor suas vontades.

Sobre a legitimidade Mitchell, Agle e Wood (1997) usam a definição de que a legitimidade é "a percepção ou pressuposição generalizada de que as ações de uma entidade são desejáveis, apropriadas ou adequadas dentro de um sistema de normas, valores, crenças e definições socialmente construídas" (SUCHMAN, 1995 apud MITCHELL; AGLE; WOOD, 1997, p. 866). Muitos autores costumam fazer uma suposição implícita de que a legitimidade e a força são atributos que estão sempre juntos, porém Mitchell, Agle e Wood (1997) concordam com Webber (1947) quando este sublinha que legitimidade e poder são atributos distintos, mas quando 
associados criam a autoridade, que seria o uso legítimo do poder, mas declara que estes dois aspectos também podem existir em separado.

Por fim, a urgência é o atributo que, quando incluso, torna o modelo de stakeholders dinâmico, porém, para que ela existasão necessárias duas condições: haver uma relação ou reivindicação de natureza sensível ao tempo; eesta relação ou reivindicação seja importante ou crítica para osstakeholders. Em razão destas condições, pode inferir que a urgência é baseada em outros dois atributos: a sensibilidade em relação ao tempo, ou seja, com quanto tempo um atraso gerencial em atender à reivindicação ou ao relacionamento se torna inaceitável para os stakeholders; e arelevância crítica, que é a importância da reivindicação ou do relacionamento com os stakeholders (MITCHELL; AGLE; WOOD, 1997). Ao associar os três atributos fazem a classificação dos stakeholders, conforme Figura 1.

Figura 1 - Modelo de Saliência dos Stakeholders

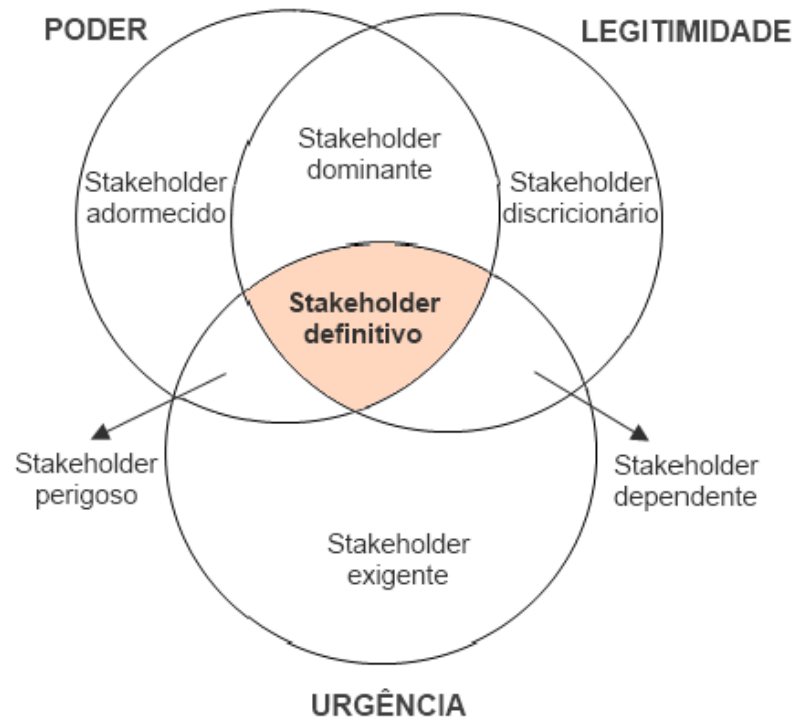

Fonte: Traduzido de Mitchell, Agle e Wood (1997, p. 872).

De acordo com o modelo, os stakeholders são divididos em três grupos de classes, a saber: latentes, em espera e definitivos. O grupo da classe dos latentes compreende os stakeholders que possuem apenas um atributo (adormecido, discricionário e exigente). O que possui apenas o atributo do poder é o stakeholder adormecido, sendo sua característica o fato ter o poder de impor sua vontade na empresa, mas sem a legitimidade e a urgência esse poder não é usado. O que possui apenas o atributo da legitimidade é o stakeholder arbitrário, que por não ter poder nem urgência os administradores não sofrem pressão para atender as suas 
necessidades, entretanto eles são o principalpúblico-alvo para receber ajudas filantrópicas. Já o que possui apenas o atributo da urgência é o stakeholder exigenteque, por não ter poder nem legitimidade, acaba sendo uma classe que incomoda os administradores, são como uma "pedra no sapato" (MITCHELL; AGLE; WOOD, 1997).

O grupo da classe dos em espera, de acordo com Mitchell, Agle e Wood (1997), é formada pelos stakeholders que possuem dois atributos (dominante, perigoso e denpedente). Quando o stakeholder possui tanto poder quanto legitimidade, ele é chamado de stakeholder dominante, pois espera e tem a atenção dos administradores devido a sua influência adquirida pelos seus atributos, mas eles não consistem nos stakeholders aos quais os administradores devem especialmente se dedicar. O stakeholder que possui legitimidade e urgência é denominado stakeholder dependente, pois, devido à falta de poder, esses stakeholders dependem de outros para que suas vontades sejam atendidas. Já aqueles stakeholders que possuem poder e urgência são denominados de stakeholder perigoso, pelo fato de que estes tendem a ser coercivos e possivelmente violentos, tornando-os literalmente perigosos para a empresa.

O terceiro e último grupo de classe é a do stakeholderdefinitivo, o qual possui os três atributos concomitantes: poder, legitimidade e urgência, e quando isso ocorre, os administradores devem dar prioridade às reivindicações destes stakeholders e atendê-los imediatamente. $\mathrm{O}$ fato comum de acontecer é o caso de um stakeholder dominante adquirir o atributo de legitimidade e tornar-se um stakeholder definitivo.

Outro modelo de análise de stakeholders foi elaborado por Friedman e Miles (2002). Os autores tem como base o modelo de Archer $(1995,1996)$ visando distinguir os diferentes tipos de stakeholders. O modelo é demonstrado no Quadro 1, no qualprevalecem dois focos em relações aos stakeholders da organização e nos intermediários institucionais (sociais e culturais) entre eles, diferenciado por lógicas situacionais. Do modelo adaptado de Archer, foram diferenciadas 4 configurações estruturais (Item A, B, C e D), aceitando-se as suas lógicas situacionais. Tem-se implícito ainda a teoria do contrato para sustentar a legalidade dos stakeholders, sendo que há contratos distintos para cada interação organização/stakeholder e estas estão associadas com cada configuração estrutural. 
Quadro 1 - Configuração e Forma de Associação Contratual e Ações Estratégicas

\begin{tabular}{|c|c|c|}
\cline { 2 - 3 } \multicolumn{1}{c|}{ Compatível } & Necessidade & Contingência \\
\hline \multirow{2}{*}{ Incompatível } & $\begin{array}{c}\text { A } \\
\text { Reconhecido explícito/implícito } \\
\text { Defensivo }\end{array}$ & $\begin{array}{c}\text { B } \\
\text { Irreconhecido implicitamente } \\
\text { Oportunista }\end{array}$ \\
& $\begin{array}{c}\text { Reconhecido explícito/implícito } \\
\text { Compromisso }\end{array}$ & $\begin{array}{c}\text { Conhum contato } \\
\text { Eliminação }\end{array}$ \\
\hline
\end{tabular}

Fonte: Traduzido de Friedman e Miles (2002, p. 9)

Os autores descrevem que contratos são entendidos como os relacionamentos incorporados com algum grau de liberdade e de acordo com pelo menos alguns dos interesses dos contratantes. O tipo ideal de contrato é incorporado sem coerção ou constrangimento e deve ser benéfico a todas as partes (embora não necessariamente igualmente benéficos). Os contratos podem ser classificados como:

(1) Contratos reconhecidos explícitos (escrito ou verbal, pode ser através de um terceiro); (2) Contratos reconhecidos implícitos (reconhecidos pelos partidos envolvidos e/ou outro significativos, tais como os governos ou reguladores ou sócios); (3) Contratos não reconhecidos implícitos (não reconhecidos pelos envolvidos, mas reconhecidos por determinado grupo como acadêmicos e ativistas); e (4) Nenhum contrato (FRIEDMAN; MILES, 2002, p. 9).

O modelo proposto por Friedman e Miles (2002) fornece uma estrutura que permite a análise de como e o porquê ocorrem às mudanças na relação organização/stakeholders. A mudança pode ocorrer em todo o sentido, sendo que geralmente ocorre: mudanças institucionais; os fatores contingentes emergem; mudança das 'idéias' das organizações; e interesses materiais de uma ou outra parte. Na sequência será tratado sobre a orientação para os stakeholders.

\subsection{ORIENTAÇÃO PARA O STAKEHOLDER}

Dentre as orientações ao stakeholders encontradas na literatura, o presente artigo concentrará nas orientações para os stakeholders primários ou visão restrita ao termo. Assim, será discutida a orientação para os clientes, para a concorrência (ou concorrentes), para os acionistas e aos colaboradores. 
Com relação à orientação para os clientes, ela se refere à importância que a empresa dá aos interesses dos clientes. Vários autores, desde meados da década de 1960, mencionam a importância dos clientes para as empresas, e que a mesma deve procurar saber, predizer e até tentar controlar as necessidades e preferências dos clientes. Empresas que seguem essa linha de pensamento tendem a investir mais na capacitação de seus colaboradores com foco no atendimento ao cliente, buscando daqueles uma atitude que agregue valor superior aos consumidores (YAU et al., 2007).

No que se refere à orientação para a concorrência, a mesma foca no conhecimento de seus concorrentes. Saber quais são suas forças e fraquezas, capacidades e estratégias, para que com isso a empresa possa "dar a resposta" para as ações dos seus competidores, sendo estes últimos definidos como os stakeholders que exercem ameaças competitivas. Uma das sugestões de alguns autores é o benchmarking, uma forma de a empresa buscar um melhor desempenho que seus concorrentes. A empresa precisa buscar conhecer, monitorar, predizer e contra-atacar as ações de seus concorrentes, mas sem deixar de ser ética, respeitando as regras do "jogo". Logo, o cuidado excessivo pode ser prejudicial. Assim, quando a empresa deixa de se preocupar com seus outros shareholders e focam todos os esforços na concorrência, pode haver consequências desastrosas, por isso deve-se buscar sempre um equilíbrio nas suas políticas de ações (YAU et al., 2007).

$\mathrm{Na}$ orientação para os acionistas, a empresa busca maximizar o retorno, buscando a lucratividade retorno sobre o capital investido e dividindo esse lucro o máximo possível com os acionistas como forma de recompensa pelo investimento (ROSS; WESTERFIELD; JAFFE, 2009). A empresa tem essa postura, muitas vezes pelo fato dos acionistas serem os 'donos' da mesma, e caso eles fiquem insatisfeitos com o andamento da empresa, podem decidir-se por vender as ações que possuem, afetando com isso o valor da empresa no mercado, assim como a sua reputação (YAU et al., 2007). Para Atkinson, Waterhouse e Wells (1997) diversas questões impulsionam o aumento do interesse sobre o retorno sobre investimento. Para os autores a pressão dos acionistas sobre os gerentes é provavelmente o driver mais influente. A importância dos acionistas tem se elevado nas economias de países desenvolvidos, esta importância levou os administradores a concentra-se na criação 
de valor como uma importante medida de desempenho (COPELAND; KOLLER; MURRIN, 2001).

Por fim, na orientação para os colaboradores, a empresa tem como foco a estabilidade e a satisfação do empregado. De acordo com Berman et al. (1999) existem evidências apontando que práticas projetadas e integradas de gestão de recursos humanos podem produzir efeitos positivos que vão além do que as iniciativas individuais específicas podem produzir. Hillman e Keim (2001) destacam que pesquisadores de estratégia têm focado a pesquisa, considerando a empresa como um ambiente institucional capaz de facilitar a aprendizagem e criar e difundir conhecimento de produzir valor para seus stakeholders. Nesse contexto institucional estão incluídos os atores, a história e o relacionamento com os stakeholders dentre estes, destacam-se os empregados.

Para isso há uma preocupação com os interesses dos empregados e busca da satisfação de suas necessidades, dentro das possibilidades da empresa. Para corroborar essa visão, estudos relacionados com a gestão de pessoas indicam que funcionários satisfeitos com seus trabalhos têm uma autoestima mais elevada, o que trás uma motivação maior para trabalhar, impactando diretamente na sua produtividade, eficiência e eficácia, principalmente nos colaboradores da linha de frente, que tem contado direto com os clientes, aumentando o nível de satisfação destes e consequentemente melhorando as vendas (YAU et al., 2007).

\section{PROCEDIMENTOS METODOLÓGICOS}

A presente pesquisa consistiu-sena replicação do estudo realizado por Yau et al. (2007) com empresas chinesas, publicado no European Journal of Marketing sob o título Developing a Scale for Stakeholder Orientation. A pesquisa realizada foi um estudo descritivo de caráter quantitativo com o objetivo de fazer a validação inicial da escala que determina a orientação aos clientes, concorrentes, acionistas e funcionários (Quadro 2). 
Quadro 2 - Escala Orientação para os stakeholders

\begin{tabular}{|c|}
\hline QUESTÃO: \\
\hline 01 Estratégias competitivas são baseadas nas necessidades dos clientes. \\
\hline 02 A satisfação do cliente é avaliada sistematicamente e com freqüência. \\
\hline $\begin{array}{l}03 \text { Nosso comprometimento em atender as necessidades dos clientes é monitorada } \\
\text { rigorosamente. }\end{array}$ \\
\hline 040 serviço de pós-venda é tratado com atenção. \\
\hline 05 Nossos objetivos e estratégias são dirigidos para a criação da satisfação do cliente. \\
\hline 06 Os vendedores compartilham informações sobre os competidores. \\
\hline $07 \mathrm{~A}$ alta gerência discute regularmente sobre as forças e fraquezas dos competidores. \\
\hline 08 Nós respondemos prontamente as ações dos concorrentes \\
\hline 09 Os clientes são o foco quando temos uma oportunidade de vantagem competitiva. \\
\hline 10 Nossos objetivos são dirigidos em estabelecer a saúde financeira de nossos acionistas. \\
\hline 11 Os administradores seniores se reúnem regularmente com os acionistas. \\
\hline 12 Nós comparamos regularmente o valor de nossas ações com os da concorrência. \\
\hline 13 Nós regularmente nos dirigimos publicamente aos nossos acionistas. \\
\hline $\begin{array}{l}14 \text { Temos administradores designados especialmente para atender aos interesses dos } \\
\text { acionistas. }\end{array}$ \\
\hline $\begin{array}{l}15 \text { Nos temos avaliações regulares com os membros da diretoria onde discutimos as } \\
\text { necessidades dos funcionários. }\end{array}$ \\
\hline 16 Nós temos reuniões regulares entre diretoria e funcionários. \\
\hline $\begin{array}{l}17 \text { Como administrador, eu tento identificar os reais sentimentos dos meus funcionários em } \\
\text { relação às suas funções. }\end{array}$ \\
\hline $\begin{array}{l}18 \text { Nós pesquisamos com os funcionários ao menos uma vez ao ano, para avaliar suas } \\
\text { atitudes perante seus trabalhos. }\end{array}$ \\
\hline
\end{tabular}

A metodologia quantitativa pode ser definida como a "descrição objetiva, sistemática e quantitativa do conteúdo manifesto da comunicação" (LAKATOS; MARCONI, 2008, p. 283). Complementando, Cooper e Schindler (2003) apresenta que o estudo descritivo serve para três tarefas: descrever fenômenos ou características associadas com a população - alvo; estimar proporções de uma população que tenham essas características; e descobrir associações entre diferentes variáveis. A presente pesquisa visou descrever, por meio da pesquisa quantitativa, o grau de orientação para os stakeholders das empresas de capital aberto, e se a adoção da orientação aos stakeholders influencia no desempenho das empresas.

No que tange a população da pesquisa está composta por 78 empresas de capital aberto sediadas na região Sul do Brasil (Paraná, Santa Catarina e Rio Grande do Sul) relacionadas no sítio da BM\&FBOVESPA (2010) (www.bmfbovespa.com.br). Num primeiro momento foi feito o contado telefônico com as 78 empresas delimitadas, com oito empresas não foi possível fazer o contato 
(não atenderam ao telefonema nas duas tentativas de contato), três justificaram que retornariam a ligação (deste modo foi passado o contato telefônico e eletrônico, mas não houve retorno). Aos outros que não descartaram responder o questionário, foi encaminhada uma mensagem eletrônica contendo o link para o questionário. 0 primeiro retorno foi composto por 7 questionários e após 15 dias foi efetuado novo contato, o qual retornaram mais 2 questionários. Assim, a presente pesquisa adotou a escolha da amostra por acessibilidade dos respondentes, haja vista que houve retorno de 9 questionários, os quais foram tabulados e serão apresentados na seção quatro.

A coleta de dados foi feita através de um survey nas empresasselecionadas como amostra. Num primeiro momento houve um contato telefônico para explicar os propósitos da pesquisa e solicitar a colaboração na mesma. Em caso afirmativo, foi encaminhado por meio eletrônico (e-mail) uma mensagem contendo o link para o questionário referente à pesquisa, que era composto por 28 questões fechadas, do tipo escalade Likert variando em cinco pontos, de 1 a 5 , sendo 18 acerca da orientação aos stakeholders (Quadro 2), e 10 com relação ao desempenho da empresa.

Em relação a análise de dados, foi feita uma análise de correlação entre as variáveis, sendo que sua interpretação dependeu do valor numérico e seu sinal. Quanto mais próximo de -1 ou de +1 for o valor $r$ da correlação, maior será a correlação linear (positiva ou negativa) entre as variáveis $x$ e $y$. Graficamente, têmse os seguintes casos para os valores de $r$. correlação perfeita positiva $(r=1)$; correlação perfeita negativa $(r=-1)$; correlação linear positiva $(0<r<1)$; correlação linear negativa $(-1<r<0)$; e correlação linear nula $(r=0)$. A correlação positiva é quando $x$ e $y$ tendem a variar no mesmo sentido, e a correlação negativa é quando elas tendem a variar em sentidos opostos.

O principal limitante da pesquisa foi a dificuldade de obter as respostas para os questionários, visto que muitas empresas consideram que as informações que serão coletadas são de caráter confidencial e tendem a não responder o questionário, embora tenha sido enfatizado o caráter acadêmico, bem como o sigilo dos dados e das respostas às empresas. 


\section{APRESENTAÇÃO DOS RESULTADOS E ANÁLISE}

As tabelas que seguem, contém a tabulação com as respostas de cada empresa, suas médias e a média de cada item das orientações para stakeholders. Foram garantidas as identidades das empresas, conforme assinalado nos procedimentos metodológicos. A Tabela 1 apresenta o grau de orientação paraos clientes das empresas respondentes.

Tabela 1 - Grau de Orientação para os Clientes

\begin{tabular}{|c|c|c|c|c|c|c|c|c|c|c|}
\hline $\begin{array}{c}\text { EMPRESA } \\
\text { QUESTÃO }\end{array}$ & $\boldsymbol{A}$ & $\boldsymbol{B}$ & $\boldsymbol{C}$ & $\boldsymbol{D}$ & $\boldsymbol{E}$ & $\boldsymbol{F}$ & $\boldsymbol{G}$ & $\boldsymbol{H}$ & $\boldsymbol{I}$ & $\begin{array}{c}\text { MEDIA } \\
\text { ITEM }\end{array}$ \\
\hline $\mathbf{1}$ & 5 & 4 & 5 & 5 & 4 & 4 & 4 & 5 & 5 & $\mathbf{4 , 5 6}$ \\
\hline $\mathbf{2}$ & 5 & 5 & 5 & 5 & 3 & 5 & 5 & 5 & 5 & $\mathbf{4 , 7 8}$ \\
\hline $\mathbf{3}$ & 5 & 5 & 5 & 4 & 4 & 5 & 5 & 5 & 5 & $\mathbf{4 , 7 8}$ \\
\hline $\mathbf{4}$ & 5 & 5 & 5 & 5 & 4 & 5 & 5 & 5 & 5 & $\mathbf{4 , 8 9}$ \\
\hline $\mathbf{5}$ & 4 & 4 & 5 & 5 & 4 & 5 & 5 & 5 & 5 & $\mathbf{4 , 6 7}$ \\
\hline MÉDIA EMPRESA & $\mathbf{4 , 8}$ & $\mathbf{4 , 6}$ & $\mathbf{5 , 0}$ & $\mathbf{4 , 8}$ & $\mathbf{3 , 8}$ & $\mathbf{4 , 8}$ & $\mathbf{4 , 8}$ & $\mathbf{5 , 0}$ & $\mathbf{5 , 0}$ & $\mathbf{4 , 7 3}$ \\
\hline
\end{tabular}

Fonte: Elaborado pelos autores a partir dos dados coletados.

A partir da Tabela 1 pode constatar a ocorrência de alta média de preocupação que as empresas têm em relação ao atendimento das necessidades de seus clientes perfaz 4,73 numa escala de 1 a 5 , sendo esta média a maior entre as orientações. É possível a partir destas notas que em geral as empresas alinham discurso e ações que visem os clientes, tornando uma estratégia competitiva na organização, como era esperado.

Ainda, no que tange a Tabela 1 , pode destacar os seguintes desvios: a empresa $E$ identificou a questão 2 como neutro, pontuando com nota 3. Isso demonstra que embora as empresas possuam uma orientação alta aos clientes, em algum momento uma empresa possui estratégia diferenciada e menos orientada ao cliente, mesmo que em menor frequência e força.

$\mathrm{Na}$ sequência foi perguntado sobre a orientação para os concorrentes e os resultados são demonstrados na Tabela 2. 
Tabela 2 - Grau de Orientação para os Concorrentes

\begin{tabular}{|c|c|c|c|c|c|c|c|c|c|c|}
\hline $\begin{array}{c}\text { EMPRESA } \\
\text { QUESTÃO }\end{array}$ & $\boldsymbol{A}$ & $\boldsymbol{B}$ & $\boldsymbol{C}$ & $\boldsymbol{D}$ & $\boldsymbol{E}$ & $\boldsymbol{F}$ & $\boldsymbol{G}$ & $\boldsymbol{H}$ & $\boldsymbol{I}$ & $\begin{array}{c}\text { MEDIA } \\
\text { ITEM }\end{array}$ \\
\hline $\mathbf{6}$ & 3 & 4 & 5 & 5 & 5 & 4 & 4 & 5 & 5 & $\mathbf{4 , 4 4}$ \\
\hline $\mathbf{7}$ & 3 & 4 & 5 & 4 & 5 & 3 & 4 & 5 & 5 & $\mathbf{4 , 2 2}$ \\
\hline $\mathbf{8}$ & 3 & 4 & 5 & 4 & 4 & 4 & 4 & 5 & 5 & $\mathbf{4 , 2 2}$ \\
\hline $\mathbf{9}$ & 5 & 5 & 5 & 5 & 5 & 5 & 5 & 5 & 5 & $\mathbf{5 , 0 0}$ \\
\hline MÉDIA EMPRESA & $\mathbf{3 , 5}$ & $\mathbf{4 , 3}$ & $\mathbf{5 , 0}$ & $\mathbf{4 , 5}$ & $\mathbf{4 , 8}$ & $\mathbf{4 , 0}$ & $\mathbf{4 , 3}$ & $\mathbf{5 , 0}$ & $\mathbf{5 , 0}$ & $\mathbf{4 , 4 7}$ \\
\hline
\end{tabular}

Fonte: Elaborado pelos autores a partir dos dados coletados.

Embora a média atingida pelas empresas seja a menor entre todas as orientações, ela ainda teve um elevado índice, pois ficou em 4,47. Este índice mostra que a orientação para os concorrentes em geral é considerada como forte e prioritária, embora a Empresa A seja uma das empresas que não prioriza a sua estratégia na orientação para os concorrentes, tendo em vista que a sua média ficou em 3,5, ou seja, próximo do ponto neutro (nota 3). Isso também pode demonstrar que a empresa A seja uma líder de mercado, e assim, faz com a mesma não tenha uma orientação forte para a concorrência.

Quanto ao grau de orientação aos acionistas, a Tabela 3 mostra os resultados.

Tabela 3 - Grau de Orientação para os Acionistas

\begin{tabular}{|c|c|c|c|c|c|c|c|c|c|c|}
\hline $\begin{array}{c}\text { EMPRESA } \\
\text { QUESTÃO }\end{array}$ & $\boldsymbol{A}$ & $\boldsymbol{B}$ & $\boldsymbol{C}$ & $\boldsymbol{D}$ & $\boldsymbol{E}$ & $\boldsymbol{F}$ & $\boldsymbol{G}$ & $\boldsymbol{H}$ & $\boldsymbol{I}$ & $\begin{array}{c}\text { MEDIA } \\
\text { ITEM }\end{array}$ \\
\hline $\mathbf{1 0}$ & 5 & 5 & 5 & 5 & 4 & 5 & 5 & 5 & 5 & $\mathbf{4 , 8 9}$ \\
\hline $\mathbf{1 1}$ & 4 & 4 & 5 & 5 & 3 & 4 & 5 & 5 & 5 & $\mathbf{4 , 4 4}$ \\
\hline $\mathbf{1 2}$ & 3 & 2 & 5 & 5 & 3 & 4 & 3 & 5 & 5 & $\mathbf{3 , 8 9}$ \\
\hline $\mathbf{1 3}$ & 5 & 4 & 5 & 5 & 4 & 4 & 5 & 5 & 5 & $\mathbf{4 , 6 7}$ \\
\hline $\mathbf{1 4}$ & 5 & 4 & 5 & 4 & 3 & 5 & 5 & 5 & 5 & $\mathbf{4 , 5 6}$ \\
\hline MÉDIA EMPRESA & $\mathbf{4 , 4}$ & $\mathbf{3 , 8}$ & $\mathbf{5 , 0}$ & $\mathbf{4 , 8}$ & $\mathbf{3 , 4}$ & $\mathbf{4 , 4}$ & $\mathbf{4 , 6}$ & $\mathbf{5 , 0}$ & $\mathbf{5 , 0}$ & $\mathbf{4 , 4 9}$ \\
\hline
\end{tabular}

Fonte: Elaborado pelos autores a partir dos dados coletados.

Pode verificar por meio da Tabela 3, que embora as empresas se preocupem muito com os acionistas (ou pelo menos demonstram essa preocupação), principalmente com sua saúde financeira (Questão 10), porém as respostas evidenciam que as empresas estão dividindo sua atenção com os demais stakeholders. Isso pode ser o reflexo da nova estrutura da empresa, que foi comentada no início do trabalho, com o modelo corporativo de stakeholders, na qual 
Análise do grau de orientação para os Stakeholders das empresas de capital aberto da Região Sul do Brasil

todos têm a sua parcela de importância, haja vista que os stakeholders são afetados ou afetam a organização.

$\mathrm{Na}$ sequência fora perguntado sobre a orientação para os colaboradores (ou funcionários) e os resultados estão demonstrados na Tabela 4.

Tabela 4 - Grau de Orientação para os Colaboradores

\begin{tabular}{|c|c|c|c|c|c|c|c|c|c|c|}
\hline $\begin{array}{c}\text { EMPRESA } \\
\text { QUESTÃO }\end{array}$ & $\boldsymbol{A}$ & $\boldsymbol{B}$ & $\boldsymbol{C}$ & $\boldsymbol{D}$ & $\boldsymbol{E}$ & $\boldsymbol{F}$ & $\boldsymbol{G}$ & $\boldsymbol{H}$ & $\boldsymbol{I}$ & $\begin{array}{c}\text { MEDIA } \\
\text { ITEM }\end{array}$ \\
\hline $\mathbf{1 5}$ & 4 & 5 & 5 & 5 & 4 & 4 & 4 & 5 & 5 & $\mathbf{4 , 5 6}$ \\
\hline $\mathbf{1 6}$ & 4 & 5 & 5 & 5 & 5 & 3 & 4 & 5 & 5 & $\mathbf{4 , 5 6}$ \\
\hline $\mathbf{1 7}$ & 5 & 5 & 5 & 5 & 5 & 4 & 4 & 5 & 5 & $\mathbf{4 , 7 8}$ \\
\hline $\mathbf{1 8}$ & 5 & 5 & 5 & 5 & 5 & 4 & 5 & 5 & 5 & $\mathbf{4 , 8 9}$ \\
\hline MÉDIA EMPRESA & $\mathbf{4 , 5}$ & $\mathbf{5 , 0}$ & $\mathbf{5 , 0}$ & $\mathbf{5 , 0}$ & $\mathbf{4 , 8}$ & $\mathbf{3 , 8}$ & $\mathbf{4 , 3}$ & $\mathbf{5 , 0}$ & $\mathbf{5 , 0}$ & $\mathbf{4 , 6 9}$ \\
\hline
\end{tabular}

Fonte: Elaborado pelos autores a partir dos dados coletados.

A Tabela 4 mostra que a empresa se preocupa com seu colaborador, porém a sua maior preocupação (questão 18) está no controle do mesmo. Por fim, tem-se a Tabela 5, a qual sintetiza as médias das orientações propostas por Yau et al. (2007) com as respectivas notas dadas pelas empresas pesquisadas, evidenciando que o grau de orientação é alto, pois obteve uma média na escala apresentada de 4,6, ou seja $90 \%$ do máximo (1 a 5, sendo cinco o maior grau de orientação).

Tabela 5 - Grau de Orientação das Empresas para cada Stakeholder

\begin{tabular}{|c|c|c|c|c|c|c|c|c|c|c|}
\hline $\begin{array}{c}\text { EMPRESA } \\
\text { STAKEHOLDERS }\end{array}$ & $\boldsymbol{A}$ & $\boldsymbol{B}$ & $\boldsymbol{C}$ & $\boldsymbol{D}$ & $\boldsymbol{E}$ & $\boldsymbol{F}$ & $\boldsymbol{G}$ & $\boldsymbol{H}$ & $\boldsymbol{I}$ & $\begin{array}{c}\text { MEDIA } \\
\text { ITEM }\end{array}$ \\
\hline CLIENTES & 4,8 & 4,6 & 5,0 & 4,8 & 3,8 & 4,8 & 4,8 & 5,0 & 5,0 & $\mathbf{4 , 7 3}$ \\
\hline CONCORRENTES & 3,5 & 4,3 & 5,0 & 4,5 & 4,8 & 4,0 & 4,3 & 5,0 & 5,0 & $\mathbf{4 , 4 7}$ \\
\hline ACIONISTAS & 4,4 & 3,8 & 5,0 & 4,8 & 3,4 & 4,4 & 4,6 & 5,0 & 5,0 & $\mathbf{4 , 4 9}$ \\
\hline COLABORADORES & 4,5 & 5,0 & 5,0 & 5,0 & 4,8 & 3,8 & 4,3 & 5,0 & 5,0 & $\mathbf{4 , 6 9}$ \\
\hline MÉDIA EMPRESA & $\mathbf{4 , 3}$ & $\mathbf{4 , 4}$ & $\mathbf{5 , 0}$ & $\mathbf{4 , 8}$ & $\mathbf{4 , 2}$ & $\mathbf{4 , 2}$ & $\mathbf{4 , 5}$ & $\mathbf{5 , 0}$ & $\mathbf{5 , 0}$ & $\mathbf{4 , 6 0}$ \\
\hline
\end{tabular}

Fonte: Elaborado pelos autores a partir dos dados coletados.

Complementar foi possível identificar que as orientações aos concorrentes e aos acionistas foram as que mais oscilaram e ainda, que puxaram as médias das orientações para baixo. Após essa análise inicial (orientação para cada stakeholder na visão da empresa), foi feita uma correlação entre as médias das empresas em cada item em relação com as médias das empresas na orientação para seu respectivo stakeholder. O resultado dessa correlação é demonstrado na Tabela 6. 
Tabela 6 - Correlação entre Item e sua Respectiva Orientação de Stakeholder

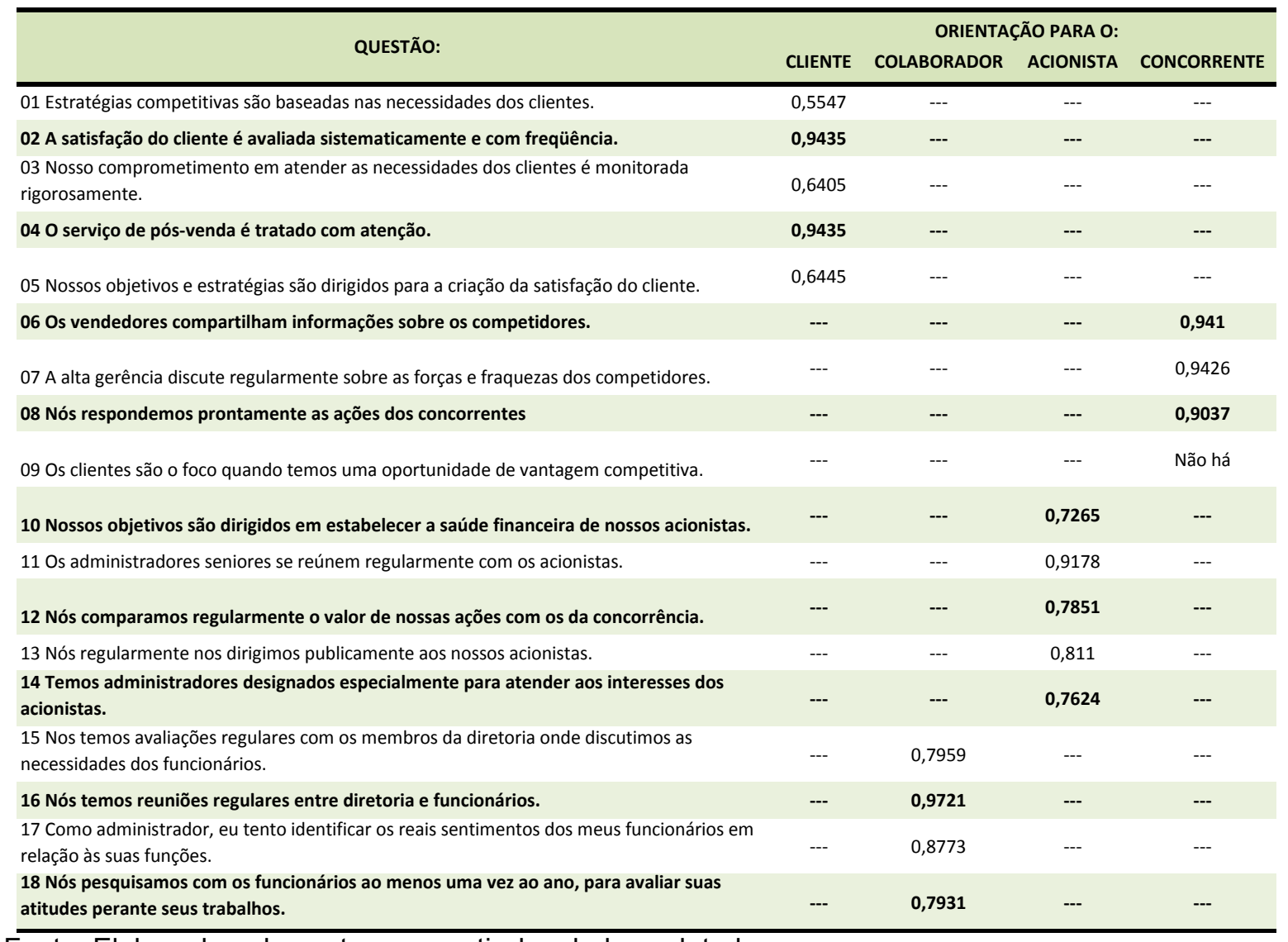

Fonte: Elaborado pelos autores a partir dos dados coletados.

Deste modo, conforme Tabela 6, a relação à orientação para os clientes teve destaque no item 2 que é a avaliação da satisfação do cliente e item 4 que é o pós-vendas são os itens que estão mais relacionados (0,9435 cada um). Percebe que as atividades desempenhadas na orientação para os clientes como atividades de rotinas.

$\mathrm{Na}$ orientação para os concorrentes, o item 9 , foco na vantagem competitiva, o cliente não teve relação com o grau de orientação aos concorrentes, pois todas as empresas apontaram esse item como de grande importância. Os outros três estão bastante relacionados com a orientação ao concorrente pelo fato de terem atingido coeficientes superiores a 0,90.

Na orientação para os acionistas, o item com maior grau de correlação foi o item 11 que contempla a reunião com os acionistas $(0,9178)$, o que mostra que quanto maior o grau de orientação para o acionista, maior é a tendência das reuniões com os mesmos, evidenciando uma aproximação e reuniões conjuntas. Em contrapartida, o item de menor correlação foi o de estabelecer a saúde financeira 
dos acionistas $(0,7265)$, devido ao fato de independente do grau de orientação ao acionista, a empresa tem um alto grau de preocupação para esse item.

Em relação à orientação para os colaboradores, o item de maior correlação foi 0 item 16 que contempla reuniões regulares entre diretoria e funcionários $(0,9721)$, o que pode inferir, pelo menos com as empresas pesquisadas, que quanto maior a propensão a realizar reuniões entre diretoria e colaboradores, maior é o grau de orientação da empresa para o colaborador. Por fim, as tabelas a seguir tratam da percepção das empresas em relação a seus desempenhos no âmbito financeiro, de mercado, satisfação de consumidor e colaborador.

Tabela 7 - Desempenho Financeiro e de Mercado

\begin{tabular}{|c|c|c|c|c|c|c|c|c|c|c|}
\hline $\begin{array}{c}\text { EMPRESA } \\
\text { QUESTÃO }\end{array}$ & $\boldsymbol{A}$ & $\boldsymbol{B}$ & $\boldsymbol{C}$ & $\boldsymbol{D}$ & $\boldsymbol{E}$ & $\boldsymbol{F}$ & $\boldsymbol{G}$ & $\boldsymbol{H}$ & $\boldsymbol{I}$ & $\begin{array}{c}\text { MÉDIA } \\
\text { ITEM }\end{array}$ \\
\hline $\mathbf{1 9}$ & 4 & 3 & 5 & 3 & 5 & 3 & 4 & 3 & 5 & $\mathbf{3 , 8 9}$ \\
\hline $\mathbf{2 0}$ & 4 & 3 & 5 & 3 & 5 & 4 & 4 & 3 & 5 & $\mathbf{4 , 0 0}$ \\
\hline $\mathbf{2 1}$ & 4 & 3 & 5 & 3 & 5 & 4 & 4 & 3 & 5 & $\mathbf{4 , 0 0}$ \\
\hline $\mathbf{2 2}$ & 5 & 4 & 4 & 3 & 3 & 2 & 4 & 5 & 5 & $\mathbf{3 , 8 9}$ \\
\hline $\mathbf{2 3}$ & 5 & 3 & 4 & 3 & 3 & 3 & 4 & 5 & 5 & $\mathbf{3 , 8 9}$ \\
\hline MÉDIA EMPRESA & $\mathbf{4 , 4}$ & $\mathbf{3 , 2}$ & $\mathbf{4 , 6}$ & $\mathbf{3 , 0}$ & $\mathbf{4 , 2}$ & $\mathbf{3 , 2}$ & $\mathbf{4 , 0}$ & $\mathbf{3 , 8}$ & $\mathbf{5 , 0}$ & $\mathbf{3 , 9 3}$ \\
\hline
\end{tabular}

Fonte: Elaborado pelos autores a partir dos dados coletados.

A Tabela 7 mostra que o desempenho financeiro e de mercado nos itens avaliados têm sido em geral parecidos (médias muito próximas), entretanto o desempenho médio das empresas teve uma grande variação (de 3,2 a 5), fato que pode ser explicado pelo fato de atuarem em diferentes ramos.

No que tange ao desempenho da satisfação dos clientes é demonstrado na Tabela 8. Esta é a categoria que obteve a maior média de desempenho para as empresas, que pode ser explicado com a média de orientação para os clientes (também a maior média das empresas quando comparada as orientações para stakeholder). Desta forma, fica notória uma orientação e preocupação forte com o atendimento das necessidades dos clientes, bem como da busca de sua satisfação.

Tabela 8 - Grau de Satisfação dos Clientes

\begin{tabular}{|c|c|c|c|c|c|c|c|c|c|c|}
\hline $\begin{array}{c}\text { EMPRESA } \\
\text { QUESTÃO }\end{array}$ & $\boldsymbol{A}$ & $\boldsymbol{B}$ & $\boldsymbol{C}$ & $\boldsymbol{D}$ & $\boldsymbol{E}$ & $\boldsymbol{F}$ & $\boldsymbol{G}$ & $\boldsymbol{H}$ & $\boldsymbol{I}$ & $\begin{array}{c}\text { MÉDIA } \\
\text { ITEM }\end{array}$ \\
\hline $\mathbf{2 4}$ & 5 & 5 & 5 & 4 & 4 & 3 & 5 & 5 & 5 & $\mathbf{4 , 5 6}$ \\
\hline $\mathbf{2 5}$ & 5 & 4 & 5 & 3 & 4 & 3 & 4 & 5 & 5 & $\mathbf{4 , 2 2}$ \\
\hline MÉDIA EMPRESA & $\mathbf{5 , 0}$ & $\mathbf{4 , 5}$ & $\mathbf{5 , 0}$ & $\mathbf{3 , 5}$ & $\mathbf{4 , 0}$ & $\mathbf{3 , 0}$ & $\mathbf{4 , 5}$ & $\mathbf{5 , 0}$ & $\mathbf{5 , 0}$ & $\mathbf{4 , 3 9}$ \\
\hline
\end{tabular}

Fonte: Elaborado pelos autores a partir dos dados coletados.

Ágora: R. Divulg. Cient., v. 21, n. 2, p. 55-75, jul./dez. 2016 (ISSNe 2237-9010) 
Em relação à satisfação dos colaboradores, assim como no desempenho financeiro e mercadológico, as empresas apresentaram uma média para os itens similares e próximas (4 e 4,11) e as médias das empresas muito variadas (3,67 a 5), o que pode indicar uma desproporção no grau de atenção que as empresas de diferentes ramos dão a seus colaboradores.

Tabela 9 - Grau de Satisfação dos Colaboradores

\begin{tabular}{|c|c|c|c|c|c|c|c|c|c|c|}
\hline $\begin{array}{c}\text { EMPRESA } \\
\text { QUESTÃO }\end{array}$ & $\boldsymbol{A}$ & $\boldsymbol{B}$ & $\boldsymbol{C}$ & $\boldsymbol{D}$ & $\boldsymbol{E}$ & $\boldsymbol{F}$ & $\boldsymbol{G}$ & $\boldsymbol{H}$ & $\boldsymbol{I}$ & $\begin{array}{c}\text { MÉDIA } \\
\text { ITEM }\end{array}$ \\
\hline $\mathbf{2 6}$ & 4 & 5 & 5 & 3 & 3 & 4 & 3 & 5 & 5 & $\mathbf{4 , 1 1}$ \\
\hline $\mathbf{2 7}$ & 4 & 3 & 5 & 4 & 5 & 4 & 3 & 3 & 5 & $\mathbf{4 , 0 0}$ \\
\hline $\mathbf{2 8}$ & 5 & 4 & 5 & 4 & 5 & 3 & 3 & 3 & 5 & $\mathbf{4 , 1 1}$ \\
\hline MÉDIA EMPRESA & $\mathbf{4 , 3}$ & $\mathbf{4 , 0}$ & $\mathbf{5 , 0}$ & $\mathbf{3 , 7}$ & $\mathbf{4 , 3}$ & $\mathbf{3 , 7}$ & $\mathbf{3 , 0}$ & $\mathbf{3 , 7}$ & $\mathbf{5 , 0}$ & $\mathbf{4 , 0 7}$ \\
\hline
\end{tabular}

Fonte: Elaborado pelos autores a partir dos dados coletados.

Por fim, a tabela 10 nos mostra a correlação entre os diversos tipos de desempenho com as diversas orientações para stakeholder.

Tabela 10 - Correlação entre Itens de Desempenho da Empresa e os Cinco Tipos de Orientações

\begin{tabular}{lccccc}
\hline \multicolumn{1}{c}{ ITEM / QUESTÃO } & \multicolumn{4}{c}{ ORIENTAÇÃO PARA O: } \\
& STAKEHOLDER & CLIENTE & COLABORADOR & ACIONISTA & CONCORRENTE \\
\hline Desempenho Financeiro e de Mercado & 0,3112 & 0,0964 & 0,3225 & 0,2462 & 0,2288 \\
Satisfação do Consumidor & 0,4896 & 0,3308 & 0,2739 & 0,3243 & 0,5456 \\
Satisfação do Colaborador & 0,3041 & 0,0224 & 0,3684 & 0,0913 & 0,4734 \\
Desempenho da Empresa & 0,4316 & 0,1808 & 0,371 & 0,2609 & 0,485 \\
\hline
\end{tabular}

Fonte: Elaborado pelos autores a partir dos dados coletados.

Vários fatos curiosos podem ser observados nessa Tabela 10, como por exemplo, a correlação é mais positiva entre a satisfação do colaborador com a orientação para a concorrência, do que a orientação ao colaborador; ou então a satisfação do consumidor está mais relacionada à orientação para os acionistas do que para o próprio consumidor. Assim, pode destacar que não existe de fato um alinhamento estratégico voltado ao atendimento dos itens levantados na Tabela 10, baseado na sua importância e similaridade dos temas pela empresa, haja vista que a correlação não apresenta nexo com os temas convergentes.

Com base nos dados divulgados ao longo do item resultados, pode inferir que as empresas pesquisadas apresentaram um alto grau de orientação para stakeholders, pois a média das empresas foi de 4,6 pontos numa escala que variava 
de 1 a 5 pontos. Outro dado que podemos inferir é o de que não há uma forte correlação entre a orientação para stakeholders e o desempenho da empresa, porém essa falta de correlação pode ser causada por várias variáveis que não puderam ser eliminadas, ou pelo menos diminuídas, por causa do tamanho da amostra. Algumas das variáveis que podem ter afetado o resultado são: as diferenças nos ramos de atividade como o produto oferecido, a percepção do cliente daquele produto, a concorrência daquele ramo; o tamanho das empresas; a sua localização; abrangência de mercado; entre outros.

Entretanto verifica-se algumas similaridades com o estudo de Yauet al.(2007), na qual esta pesquisa foi apoiada, como: a orientação para o cliente teve uma correlação mais positiva com os itens 2 (avaliação frequente da satisfação do cliente) e 4 (pós-venda); na orientação para acionistas, a sequência dos itens mais aos menos correlacionados houve apenas a inversão dos itens 12 (comparação das ações) e 14 (possuir administrador exclusivo encarregado da relação com o investidor); e, embora não tão acentuada as diferenças, a sequência dos itens mais aos menos correlacionados com a orientação para o colaborador foram a mesma. Porém, na orientação para concorrentes não houve semelhanças, além do fato do item 9 (foco da vantagem competitiva) não possuir correlação, pois todos os entrevistados deram a mesma resposta.

\section{CONSIDERAÇÕES}

$\mathrm{Na}$ atualidade, as pessoas envolvidas no mercado empresarial estão cada vez mais preocupadas com os assuntos relacionados aos seus stakeholders (YAU et al., 2007). Esse fato fica mais evidente quando Donaldson e Preston (1995) comparam o modelo atual corporativo de stakeholder com o modelo empresarial convencional de entrada-saída (input-output), evidenciando que as relações entre as empresas e seus stakeholders se tornaram mais complexas e interdependentes.

A presente pesquisa teve como objetivo geral analisar o grau de orientação para os stakeholders das empresas de capital aberto da região sul do Brasil. Embora a pesquisa tenha apontado um alto grau de orientação para os stakeholders, não há como generalizar essa afirmativa, tendo em vista que: a partir da literatura que vise a determinação de uma amostra é indicado respeitar os seguintes critérios para uma 
confiabilidade, sendo necessário erro amostral de no máximo $5 \%$, nível de confiança igual ou maior que $95 \%$ e uma busca da homogeneização dos estratos e critérios de localização, participação, dentre outros (COOPER; SCHINDLER, 2003; FAVERO et al., 2009). Desta forma, a partir destes critérios a amostra necessária seria de 65 empresas participantes (com população de 78 empresas) e se deduzir as empresas que afirmaram categoricamente o não interesse na participação da pesquisa (8 afirmaram no primeiro contato e 3 no segundo contato) reduz a população para 67 , sendo necessária 58 empresas. Porém foram retornados apenas 9 questionários respondidos, indicando assim uma quantidade considerado como não significativa.

Ao fazer a análise dos dados observou-se que, pelo menos entre as empresas entrevistadas, há uma grande preocupação com a relação com os seus stakeholders, nessa pesquisa em especial com os clientes, consumidores, acionistas e colaboradores. Enquanto que a correlação entre a orientação para stakeholders e o desempenho da empresa, por motivos diversos, não foi corroborada. Também não foi verificada a correlação entre o desempenho da empresa e a orientação para um dos quatro stakeholders selecionados para a pesquisa, o que mais se aproximou foi a relação entre a orientação para colaboradores e 2 dos 3 desempenhos específicos, satisfação dos clientes $(0,5456)$, satisfação dos colaboradores $(0,4738)$ e o desempenho geral $(0,4850)$.

Porém, a tese inicial não pode ser descartada, visto que um dos possíveis motivos da descaracterização da hipótese pode ser o baixo número de retorno das empresas contatadas. Para eliminar essa dúvida e qualquer viés recomenda-se como pesquisa futura, realizar a tentativa de uma abrangência maior do universo amostral, para que assim a tese inicial possa ser refutada ou corroborada.

\section{REFERÊNCIAS}

ALMEIDA, G. S.; FONTES FILHO, J.; MARTINS, H. Identificando stakeholders para formulação de estratégias organizacionais. In: XXIV Encontro da Associação Nacional de Pós-Graduação e Pesquisa em Administração, Florianópolis, Santa Catarina, 2000.

ATKINSON, A. A.; WATERHOUSE, J. H.; WELLS, R. B. A stakeholder approach to strategic performance measurement. Sloan Management Review, v. 38, n. 3, p. 2537, 1997. 
BERMAN, S. L.; WICKS, A. C.; KOTHA, S.; JONES, T. M. Does stakeholder orientation matter? The relationship between stakeholder management models and firm financial performance. Academy of Management Journal, v. 42, n. 5, p. 488506, 1999.

BM\&FBOVESPA. Empresas listadas. Disponível em:

$<$ http://www.bmfbovespa.com.br/cias-listadas/Empresas-

Listadas/BuscaEmpresaListada.aspx?idioma=pt-br> Acesso em: 22 jun. 2010.

CINTRA, R. F.; AMÂNCIO-VIEIRA, S. F.; COSTA, B. K. Stakeholder theory e institucionalismo sociológico: complementações para análise do turismo local de Londrina/PR. In: XXXVIII Encontro da Associação Nacional de Pós-Graduação e Pesquisa em Administração, Rio de Janeiro, 2014.

CINTRA, R. F.; AMÂNCIO-VIEIRA, S. F.; SUZUKI, T. M.; COSTA, B. K. Stakeholder theory: análise nos periódicos brasileiros a partir da bibliometria. Revista

Portuguesa e Brasileira de Gestão, v. 13, n. 4, p. 43-55, 2014.

CINTRA, R. F.; AMÂNCIO-VIEIRA, S. F.; GONCALVES, L. P.; COSTA, B. K. Turismo local de Londrina-PR: análise a partir da teoria do stakeholder. Contextus Revista Contemporânea de Economia e Gestão, v. 13, n. 2, p. 149-175, 2015.

COOPER, D. R.; SCHINDLER, P. S. Métodos de pesquisa em administração. 7.ed. Porto Alegre: Bookman, 2003.

COPELAND, T.; KOLLER, T.; MURRIN, J. Valuation: measuring and managing the value of companies. 3. ed. Mackinsey \& Company, Inc. 2001.

DONALDSON, T.; PRESTON, L. E. The stakeholder theory of the corporation: concepts, evidences, and implications. Academy of Management Review, v. 20, n. 1, p. 65-91, 1995.

FAVERO, L. P. et al. Análise de dados: modelagem multivariada para tomada de decisões. Rio de Janeiro: Campus, 2009.

FREEMAN, E. R. Strategic management: a stakeholder approach. Boston: Pitman, 1984.

FREEMAN, R. E. et al. Stakeholder theory: the state of the art. Cambrige: University Press, 2010.

FRIEDMAN, A. L.; MILES, S. Developing Stakeholder Theory. Journal of Management Studies, v. 39, n. 1, 2002.

FRIEDMAN, A. L.; MILES, S. Stakeholder: theory and practice. New York: Oxford University Press, 2006. 
HILLMAN, A. J.; KEIM, G. D. Shareholder value, stakeholder management, and social issues: what's the bottom line? Strategic Management Journal, v. 22, n. 2, p. 125-139, 2001.

LAKATOS, E. M.; MARCONI, M. A. Metodologia Científica. 5.ed. São Paulo: Atlas, 2008.

LAPLUME, A. O.; SONPAR, K.; LITZ, R. A. Stakeholder theory: reviewing a theory that moves us. Journal of Management, v. 34, n. 6, p. 1152-1189, 2008.

MILES, S. Stakeholder theory classification: a theoretical and empirical evaluation of definitions. Journal Business Ethics, p. 1-23, 2015.

MITCHELL, R. K.; AGLE, B. R.; WOOD, D. J. Towards a Theory of Stakeholder Identification and Salience: defining the principle of who and what really counts. Academy of Management Review, v. 22, n. 4, p. 853-886, Oct. 1997.

REED, M. S.; GRAVES, A.; DANDY, N.; POSTHUMUS, H.; HUBACEK, K.; MORRIS, J.; PRELL, C.; QUINN, C. H.; STRINGER, L. C.Who's in and why? A typology of stakeholder analysis methods for natural resource management. Journal of Environmental Management, v. 90, p. 1933-1949, 2009.

ROSS, S. A; WESTERFIELD, R. W. ;JAFFE, J. F. Administração financeira. 2.ed. São Paulo: Atlas 2009.

YAU, O. H. M. et al. Developing a Scale for Stakeholder Orientation. European Journal of Marketing, v. 41, n. 11-12, p. 1306-1327, 2007.

Artigo recebido em: 26/12/2016

Artigo aprovado em: 02/01/2017 